\title{
FREE RESOLUTIONS OVER COMMUTATIVE KOSZUL ALGEBRAS
}

\author{
Luchezar L. Avramov, Aldo Conca, and Srikanth B. Iyengar
}

Abstract. For $R=Q / J$ with $Q$ a commutative $\mathbb{N}$-graded algebra over a field and $J \neq 0$, we relate the slopes of the minimal resolutions of $R$ over $Q$ and of $k=R / R_{+}$ over $R$. When $Q$ and $R$ are Koszul and $J_{1}=0$ we prove $\operatorname{Tor}_{i}^{Q}(R, k)_{j}=0$ for $j>2 i \geq 0$, and also for $j=2 i$ when $i>\operatorname{dim} Q-\operatorname{dim} R$ and $\operatorname{pd}_{Q} R$ is finite.

Let $K$ be a field and $Q$ a commutative $\mathbb{N}$-graded $K$-algebra with $Q_{0}=K$. Each graded $Q$-module $M$ with $M_{j}=0$ for $j \ll 0$ has a unique up to isomorphism minimal graded free resolution, $F^{M}$. The module $F_{i}^{M}$ has a basis element in degree $j$ if and only if $\operatorname{Tor}_{i}^{Q}(k, M)_{j} \neq 0$ holds, where $k=Q / Q_{+}$for $Q_{+}=\bigoplus_{j \geqslant 1} Q_{j}$.

Important structural information on $F^{M}$ is encoded in the sequence of numbers

$$
t_{i}^{Q}(M)=\sup \left\{j \in \mathbb{Z} \mid \operatorname{Tor}_{i}^{Q}(k, M)_{j} \neq 0\right\} .
$$

It is distilled through the notion of Castelnuovo-Mumford regularity, defined by

$$
\operatorname{reg}_{Q} M=\sup _{i \geqslant 0}\left\{t_{i}^{Q}(M)-i\right\} .
$$

One has $\operatorname{reg}_{Q} k \geq 0$, and equality means that $Q$ is Koszul; see, for instance, [17].

When the $K$-algebra $Q$ is finitely generated, every finitely generated graded $Q$ module $M$ has finite regularity if and only if $Q$ is a polynomial ring over some Koszul algebra, see [6]; by contrast, the slope of $M$ over $Q$, defined to be the real number

$$
\operatorname{slope}_{Q} M=\sup _{i \geqslant 1}\left\{\left(t_{i}^{Q}(M)-t_{0}^{Q}(M)\right) / i\right\},
$$

is always finite; see Corollary 1.3. Following Backelin [7], we set Rate $Q=\operatorname{slope}_{Q} Q_{+}$ and note that one has Rate $Q \geq 1$, with equality if and only if $Q$ is Koszul.

Main Theorem. If $Q$ is a finitely generated commutative Koszul $K$-algebra and $J$ a homogeneous ideal with $0 \neq J \subseteq\left(Q_{+}\right)^{2}$, then for $R=Q / J$ and $c=$ Rate $R$ one has

(1) $\max \{c, 2\} \leq \operatorname{slope}_{Q} R \leq c+1$, with $c<\operatorname{slope}_{Q} R$ when $\operatorname{pd}_{Q} R$ is finite.

(2) $t_{i}^{Q}(R)=i(c+1)$ for some $i \geq 1$ implies $i \leq \operatorname{rank}_{k}\left(J / Q_{+} J\right)_{c+1}$ and the equalities

$$
t_{h}^{Q}(R)=h(c+1) \quad \text { for } 1 \leq h \leq i .
$$

(3) $t_{i}^{Q}(R)<i(c+1)$ holds for all $i>\operatorname{dim} Q-\operatorname{dim} R$ when $\operatorname{pd}_{Q} R$ is finite.

(4) $\operatorname{reg}_{Q} R \leq c \cdot \operatorname{pd}_{Q} R$; when $Q$ is a standard graded polynomial ring, equality holds if and only if $J$ is generated by a $Q$-regular sequence of forms of degree $c+1$.

Received by the editors May 25, 2010.

2000 Mathematics Subject Classification. Primary 13D40, 16 S37.

Key words and phrases. Koszul algebra, Castelnuovo-Mumford regularity, slope of modules.

Research partly supported by NSF grants DMS 0803082 (LLA) and 0602498 (SBI). 
The result is new even in the case of a polynomial ring $Q$, where a related statement was initially proved by using Gröbner bases; see 5.3.

The theorem is proved in Section 4. Its assertions have very different underpinnings: The inequalities in (1) come from results in homological algebra, established in Section 1 with no finiteness hypotheses on $Q$. The remaining statements are deduced from results about small homomorphism $Q \rightarrow R$, proved in Section 3 by using delicate properties of commutative noetherian rings.

Our results single out classes of quadratic algebras that contain the class of Koszul algebras or are subsumed in it. The relations between them are clarified in Section 6 .

Much of the discussion in the body of the paper concerns the general problem of relating properties of the numbers slope $Q$, ,lope $_{Q} R$, and slope $_{R} M$, when $Q \rightarrow R$ is a homomorphism of graded $K$-algebras and $M$ is a graded module defined over $R$. Slopes are also studied in [10], and are related to invariants introduced in [2].

The essence of our results is a comparison of two types of degrees, ones arising from homological considerations, the others induced by internal gradings of the objects under study. In constructions involving two or more gradings the index referring to an internal degree always appears last. When $y$ is a homogeneous element of a bigraded object, $|y|$ denotes the homological degree and $\operatorname{deg}(y)$ the internal degree.

The proofs presented below involve various homological constructions that are well documented in the case of commutative local rings and their local homomorphisms, but for which graded analogs may be difficult to find in the literature. We occasionally state results in the graded context with references to sources dealing with the local situation. We have verified - and invite readers to follow suit - that in these instances internal degrees can be tracked through all the arguments involved.

\section{Slopes of graded modules}

In this section $\varphi: Q \rightarrow R$ is a surjective homomorphism of graded $K$-algebras, and $M$ is a graded $R$-module with $M_{j}=0$ for all $j \ll 0$; we set $J=\operatorname{Ker} \varphi$.

We recall a classical change-of-rings spectral sequence of Cartan and Eilenberg.

1.1. By $[12$, Ch. XVI, $§ 5]$, there exists a spectral sequence of trigraded $k$-vector spaces

$$
{ }^{r} \mathrm{E}_{p, q, j} \underset{p}{\Longrightarrow} \operatorname{Tor}_{p+q}^{Q}(k, M)_{j} \quad \text { for } \quad r \geq 2,
$$

with differentials acting according to the pattern

$$
{ }^{r} \mathrm{~d}_{p, q, j}:{ }^{r} \mathrm{E}_{p, q, j} \rightarrow{ }^{r} \mathrm{E}_{p-r, q+r-1, j} \quad \text { for } \quad r \geq 2,
$$

with second page of the form

$$
{ }^{2} \mathrm{E}_{p, q, j} \cong \bigoplus_{j_{1}+j_{2}=j} \operatorname{Tor}_{p}^{R}(k, M)_{j_{1}} \otimes_{k} \operatorname{Tor}_{q}^{Q}(k, R)_{j_{2}}
$$

and with edge homomorphisms

$$
\operatorname{Tor}_{i}^{Q}(k, M)_{j} \rightarrow{ }^{\infty} \mathrm{E}_{i, 0, j}={ }^{i+1} \mathrm{E}_{i, 0, j} \hookrightarrow{ }^{2} \mathrm{E}_{i, 0, j} \cong \operatorname{Tor}_{i}^{R}(k, M)_{j}
$$

equal to the canonical homomorphisms of $k$-vector spaces

$$
\operatorname{Tor}_{i}^{\varphi}(k, M)_{j}: \operatorname{Tor}_{i}^{Q}(k, M)_{j} \rightarrow \operatorname{Tor}_{i}^{R}(k, M)_{j} .
$$

For all $r, p$, and $q$ we set $\sup ^{r} \mathrm{E}_{p, q, *}=\sup \left\{\left.j \in \mathbb{Z}\right|^{r} \mathrm{E}_{p, q, j} \neq 0\right\}$. 
The proof of the next result is based on an analysis of the convergence of the preceding change-of-rings spectral sequence on the line $q=0$.

Proposition 1.2. When $J \neq Q J_{1}$ holds there are inequalities

$$
\operatorname{slope}_{R} M \leq \max \left\{\operatorname{slope}_{Q} M, \sup _{i \geqslant 1}\left\{\frac{t_{i}^{Q}(R)-1}{i}\right\}\right\} \leq \max \left\{\operatorname{slope}_{Q} M, \text { slope }_{Q} R\right\} \text {. }
$$

Proof. If $t_{i}^{Q}(R)$ or $t_{i}^{Q}(M)$ is infinite for some $i \geq 0$, then so are both maxima above, hence there is nothing to prove. Thus, we may assume that $t_{i}^{Q}(R)$ and $t_{i}^{Q}(M)$ are finite for every $i \geq 0$; in this case the second inequality is clear. Let $m$ denote the middle term in the inequalities above. Using the equality $t_{0}^{Q}(M)=t_{0}^{R}(M)$, we get

$$
\begin{aligned}
t_{i}^{Q}(M) & \leq m i+t_{0}^{R}(M) ; \\
t_{i}^{Q}(R) & \leq m i+1 .
\end{aligned}
$$

For $i \geq 0$ and $r \geq 2$, from formulas (1.1.2) and (1.1.3) one gets exact sequences

$$
0 \longrightarrow{ }^{r+1} \mathrm{E}_{i, 0, j} \longrightarrow{ }^{r} \mathrm{E}_{i, 0, j} \stackrel{{ }^{r} \mathrm{~d}_{i, 0, j}}{\longrightarrow}{ }^{r} \mathrm{E}_{i-r, r-1, j} .
$$

We set up a primary induction on $i$ and a secondary, descending one, on $r$ to prove

$$
\sup ^{r} \mathrm{E}_{i, 0, *} \leq m i+t_{0}^{R}(M) \text { and } i+1 \geq r \geq 2 .
$$

In view of (1.1.3), the validity of (1.2.4) $)_{i, 2}$ is the assertion of the proposition.

The basis of the primary induction, for $i=1$, comes from (1.1.4) and (1.2.1) 1 .

Fix an integer $i \geq 2$ and assume that (1.2.4) $)_{i^{\prime}, r}$ holds for $i^{\prime}<i$. Formulas (1.1.4) and $(1.2 .1)_{i}$ imply $(1.2 .4)_{i, i+1}$. Fix $r \in[2, i]$ and assume that $(1.2 .4)_{i, r^{\prime}}$ holds for $i+1 \geq r^{\prime}>r$. The first relation in the following chain

$$
\begin{aligned}
\sup ^{r} \mathrm{E}_{i, 0, *} & \leq \max \left\{\sup ^{r+1} \mathrm{E}_{i, 0, *}, \sup ^{r} \mathrm{E}_{i-r, r-1, *}\right\} \\
& \leq \max \left\{m i+t_{0}^{R}(M), \sup ^{r} \mathrm{E}_{i-r, r-1, *}\right\} \\
& \leq \max \left\{m i+t_{0}^{R}(M), \sup ^{2} \mathrm{E}_{i-r, r-1, *}\right\} \\
& =\max \left\{m i+t_{0}^{R}(M), t_{i-r}^{R}(M)+t_{r-1}^{Q}(R)\right\} \\
& \leq \max \left\{m i+t_{0}^{R}(M),\left(m(i-r)+t_{0}^{R}(M)\right)+(m(r-1)+1)\right\} \\
& =\max \left\{m i+t_{0}^{R}(M), m i+t_{0}^{R}(M)-(m-1)\right\} \\
& \leq m i+t_{0}^{R}(M)
\end{aligned}
$$

comes from the exact sequence (1.2.3). The second one holds by $(1.2 .4)_{i, r+1}$, the third because ${ }^{r} \mathrm{E}_{i-r, r-1, *}$ is a subfactor of ${ }^{2} \mathrm{E}_{i-r, r-1, *}$, the fourth by (1.1.3), the fifth by $(1.2 .4)_{i-r, 2}$ and $(1.2 .2)_{r-1}$, and the last one because $J \neq Q J_{1}$ implies $m \geq 1$.

This completes the inductive proof of the inequality $(1.2 .4)_{i, r}$.

Variants of the proposition have been known for some time, at least when $M$ is finitely generated and $R$ is standard graded; that is, $R=K\left[R_{1}\right]$ with $\operatorname{rank}_{K} R_{1}$ finite. Thus, Aramova, Bărcănescu, and Herzog in $[2,1.3]$ established the corresponding result for a related invariant, $\operatorname{rate}_{R} M=\sup _{i \geqslant 1}\left\{t_{i}^{Q}(M) / i\right\}$. They used the same spectral sequence, extending an argument of Avramov for $M=k$, see [7, p. 97]; in the latter case, the corollary below was first proved by Anick in $[1,4.2]$. 
Corollary 1.3. If $R$ is finitely generated over $K$, then for every finitely generated $R$-module $M$ one has slope $_{R} M<\infty$.

Proof. One may choose $Q$ to be a polynomial ring in finitely many indeterminates over $K$. In this case $\operatorname{Tor}_{i}^{Q}(k, R)_{*}$ and $\operatorname{Tor}_{i}^{Q}(k, M)_{*}$ are finitely generated over $k$ for each $i \geq 0$ and are zero for almost all $i$, so slope ${ }_{Q} R$ and slope $_{Q} M$ are finite.

For the next result we study the convergence of the spectral sequence 1.1 on the line $p=0$. See 3.4 and Proposition 4.1 for cases when the hypothesis on Tor ${ }^{\varphi}$ holds.

Proposition 1.4. If $M \neq 0$ and $\operatorname{Tor}_{i}^{\varphi}(k, M)$ is injective for each $i$, then one has

$$
\text { slope }_{Q} R \leq 1+s \quad \text { where } s=\sup _{i \geqslant 2}\left\{\frac{t_{i}^{R}(M)-t_{0}^{R}(M)-1}{i-1}\right\} .
$$

Proof. The hypothesis implies $t_{0}^{R}(M)>-\infty$. There is nothing to prove if $t_{i}^{Q}(M)=\infty$ for some $i$, so we assume that $t_{i}^{Q}(M)$ is finite for all $i \geq 0$. By the definition of the number $s$, the following inequalities then hold:

$$
t_{i}^{R}(M) \leq s(i-1)+1+t_{0}^{R}(M) \text { for all } i \geq 2 .
$$

It follows from (1.1.2) and (1.1.3) that for $r \geq 2$ there exist exact sequences

$$
{ }^{r} \mathrm{E}_{r, i-r+1, j} \stackrel{{ }^{r} \mathrm{~d}_{r, i-r+1, j}}{\longrightarrow}{ }^{r} \mathrm{E}_{0, i, j} \longrightarrow{ }^{r+1} \mathrm{E}_{0, i, j} \longrightarrow 0
$$

By primary induction on $i$ and secondary, descending induction on $r$, we prove

$$
\sup ^{r} \mathrm{E}_{0, i, *} \leq(s+1) i+t_{0}^{R}(M) \text { for } i+2 \geq r \geq 2 .
$$

In view of (1.1.3), the validity of (1.4.3) $)_{i, 2}$ yields the assertion of the proposition.

The injectivity of $\operatorname{Tor}^{\varphi}(k, M)$ and (1.1.4) imply ${ }^{\infty} \mathrm{E}_{p, q, *}=0$ for $q \geq 1$ and all $p$. It follows from (1.1.2) and (1.1.3) that ${ }^{n+2} \mathrm{E}_{0, i, *}$ is isomorphic to $\operatorname{Tor}_{0}^{R}(k, M)_{*}$ for $i=0$ and to 0 for $i \geq 1$, so $(1.4 .3)_{i, i+2}$ holds for all $i \geq 0$. This gives the basis of the primary induction for $i=0$ and that of the secondary induction for all $i \geq 1$.

Fix an integer $i \geq 1$ and assume that $(1.4 .3)_{i^{\prime}, r^{\prime}}$ holds for all pairs $\left(i^{\prime}, r^{\prime}\right)$ with $i^{\prime}<i$ and $i+2 \geq r^{\prime}>r$. One then has a chain of relations

$$
\begin{aligned}
\sup ^{r} \mathrm{E}_{r, i-r+1, *} & \leq \sup ^{2} \mathrm{E}_{r, i-r+1, *} \\
& =t_{r}^{R}(M)+t_{i-r+1}^{Q}(R) \\
& \leq t_{r}^{R}(M)+(s+1)(i-r+1) \\
& \leq s(r-1)+1+t_{0}^{R}(M)+(s+1)(i-r+1) \\
& =(s+1) i+(2-r)+t_{0}^{R}(M) \\
& \leq(s+1) i+t_{0}^{R}(M),
\end{aligned}
$$

where the first one holds because ${ }^{r} \mathrm{E}_{r, i-r+1, *}$ is a subfactor of ${ }^{2} \mathrm{E}_{r, i-r+1, *}$, the second by formula (1.1.3), the third by (1.4.3) $)_{i-r+2,2}$ and (1.1.3), and the fourth by (1.4.1) ${ }_{r}$. The exact sequence (1.4.2), the preceding inequalities, and (1.4.3) $)_{i, r+1}$ give

$$
\begin{aligned}
\sup ^{r} \mathrm{E}_{0, i, *} & \leq \max \left\{\sup ^{r+1} \mathrm{E}_{0, i, *}, \sup ^{r} \mathrm{E}_{r, i-r+1, *}\right\} \\
& \leq(s+1) i+t_{0}^{R}(M) .
\end{aligned}
$$

Hereby, the inductive proof of the inequality $(1.4 .3)_{i, r}$ is complete. 


\section{Regular elements}

Not surprisingly, the bounds obtained in the preceding section can be sharpened in cases when the minimal free resolution of $R$ or of $M$ over $Q$ is particularly simple.

Proposition 2.1. If $R=Q /(f)$ for a non-zero divisor $f \in Q_{+}$, then one has:

(1) slope $_{Q} M \leq \max \left\{\operatorname{slope}_{R} M, \operatorname{deg}(f)\right\} \quad$ with equality for $f \notin\left(Q_{+}\right)^{2}$.

(2) slope $_{R} M \leq \max \left\{\operatorname{slope}_{Q} M, \operatorname{deg}(f) / 2\right\} \quad$ with equality for $f \in Q_{+} \operatorname{Ann}_{Q} M$.

Proof. We start by noting an elementary inequality that will be invoked a couple of times: All pairs of real numbers $\left(a_{1}, a_{2}\right)$ and $\left(b_{1}, b_{2}\right)$ with positive $b_{1}$ and $b_{2}$ satisfy

$$
\frac{a_{1}+a_{2}}{b_{1}+b_{2}} \leq \max \left\{\frac{a_{1}}{b_{1}}, \frac{a_{2}}{b_{2}}\right\} \text {. }
$$

Set $d=\operatorname{deg}(f)$. The minimal graded free resolution of $R$ over $Q$ is

$$
0 \longrightarrow Q(-d) \stackrel{f}{\longrightarrow} Q \longrightarrow 0
$$

so $\operatorname{Tor}_{q}^{Q}(R, k)$ vanishes for $q \neq 0,1$, is isomorphic to $k$ for $q=0$, and to $k(-d)$ for $q=1$, so for each pair $(i, j)$ the spectral sequence 1.1 yields an exact sequence

$$
\begin{array}{r}
\operatorname{Tor}_{i+1}^{R}(k, M)_{j} \stackrel{\delta_{i+1, j}}{\longrightarrow} \operatorname{Tor}_{i-1}^{R}(k, M)_{j-d} \\
\longrightarrow \operatorname{Tor}_{i}^{Q}(k, M)_{j} \longrightarrow \operatorname{Tor}_{i}^{R}(k, M)_{j} \stackrel{\delta_{i, j}}{\longrightarrow} \operatorname{Tor}_{i-2}^{R}(k, M)_{j-d}
\end{array}
$$

The one for $i=0$ gives the following equality:

$$
t_{0}^{Q}(M)=t_{0}^{R}(M)
$$

(1) For $i \geq 1$ the middle three terms of the exact sequences (2.1.2) yield

$$
t_{i}^{Q}(M) \leq \max \left\{t_{i}^{R}(M), t_{i-1}^{R}(M)+d\right\}
$$

From (2.1.4), (2.1.3), and (2.1.1) we obtain the inequalities below:

$$
\begin{aligned}
\text { slope }_{Q} M & =\sup _{i \geqslant 1}\left\{\left(t_{i}^{Q}(M)-t_{0}^{Q}(M)\right) / i\right\} \\
& \leq \sup _{i \geqslant 1}\left\{\max \left\{\frac{t_{i}^{R}(M)-t_{0}^{R}(M)}{i}, \frac{t_{i-1}^{R}(M)-t_{0}^{R}(M)+d}{(i-1)+1}\right\}\right\} \\
& \leq \sup _{i \geqslant 2}\left\{\max \left\{\frac{t_{i}^{R}(M)-t_{0}^{R}(M)}{i}, \frac{t_{i-1}^{R}(M)-t_{0}^{R}(M)}{i-1}, d\right\}\right\} \\
& =\max \left\{\sup _{i \geqslant 1}\left\{\frac{t_{i}^{R}(M)-t_{0}^{R}(M)}{i}\right\}, d\right\} \\
& =\max \left\{\operatorname{sope}_{R} M, d\right\} .
\end{aligned}
$$

When $f \notin\left(Q_{+}\right)^{2}$ holds, the proof in $[4,3.3 .3(1)]$ of a result of Nagata, implies $\delta_{i, j}=0$ in (2.1.2), so equalities hold in (2.1.4). This and (2.1.3) give

$$
\begin{aligned}
& t_{1}^{Q}(M)-t_{0}^{Q}(M)=\max \left\{t_{1}^{R}(M)-t_{0}^{R}(M), d\right\}, \\
& t_{i}^{Q}(M)-t_{0}^{Q}(M) \geq t_{i}^{R}(M)-t_{0}^{R}(M) \text { for } i \geq 2 .
\end{aligned}
$$

The preceding relations clearly imply slope ${ }_{Q} M \geq \max \left\{\operatorname{slope}_{R} M, d\right\}$. 
(2) For $i \geq 1$ the last three terms of the exact sequences (2.1.2) yield

$$
\begin{aligned}
t_{i}^{R}(M) & \leq \max \left\{t_{i}^{Q}(M), t_{i-2}^{R}(M)+d\right\} \\
& \leq \max \left\{t_{i}^{Q}(M), t_{i-2}^{Q}(M)+d, t_{i-4}^{R}(M)+2 d\right\} \\
& \leq \cdots \leq \max _{0 \leqslant 2 h \leqslant i}\left\{t_{i-2 h}^{Q}(M)+h d\right\} .
\end{aligned}
$$

From (2.1.5), (2.1.3), and (2.1.1) we obtain the inequalities below:

$$
\begin{aligned}
\text { slope }_{R} M & =\sup _{i \geqslant 1}\left\{\left(t_{i}^{Q}(M)-t_{0}^{Q}(M)\right) / i\right\} \\
& \leq \sup _{i \geqslant 1}\left\{\max _{0 \leqslant 2 h \leqslant i}\left\{\frac{t_{i-2 h}^{Q}(M)-t_{0}^{Q}(M)+h d}{(i-2 h)+(2 h)}\right\}\right\} \\
& \leq \sup _{i \geqslant 1}\left\{\max _{0 \leqslant 2 h<i}\left\{\frac{t_{i-2 h}^{Q}(M)-t_{0}^{Q}(M)}{i-2 h}, \frac{d}{2}\right\}\right\} \\
& =\max \left\{\sup _{i \geqslant 1}\left\{\frac{t_{i}^{Q}(M)-t_{0}^{Q}(M)}{i}\right\}, \frac{d}{2}\right\} \\
& =\max \left\{\operatorname{slope}_{Q} M, d / 2\right\} .
\end{aligned}
$$

For $f \in Q_{+} \operatorname{Ann}_{Q} M$, the proof in $[4,3.3 .3(2)]$ of a result of Shamash shows that $\delta_{i, *}$ in (2.1.2) is surjective, so equalities hold in (2.1.5); in view of (2.1.3) one gets

$$
\begin{aligned}
& t_{1}^{R}(M)-t_{0}^{R}(M)=t_{1}^{Q}(M)-t_{0}^{Q}(M), \\
& t_{2}^{R}(M)-t_{0}^{R}(M)=\max \left\{t_{2}^{Q}(M)-t_{0}^{Q}(M), d\right\}, \\
& t_{i}^{R}(M)-t_{0}^{R}(M) \geq t_{i}^{Q}(M)-t_{0}^{Q}(M) \text { for } i \geq 3 .
\end{aligned}
$$

These relations clearly imply an inequality $\operatorname{slope}_{R} M \geq \max \left\{\operatorname{slope}_{Q} M, d / 2\right\}$.

\section{Small homomorphisms of graded algebras}

A homomorphism $\varphi: Q \rightarrow R$ of graded $K$-algebras is called small if the map

$$
\operatorname{Tor}_{i}^{\varphi}(k, k)_{j}: \operatorname{Tor}_{i}^{Q}(k, k)_{j} \rightarrow \operatorname{Tor}_{i}^{R}(k, k)_{j}
$$

is injective for each pair $(i, j) \in \mathbb{N} \times \mathbb{Z}$; see 3.4 for examples.

Although conditions (i) and (ii) in the next theorem are numerical, the proof that (i) implies (ii) pivots on nontrivial properties of the bigraded $k$-algebra $\operatorname{Tor}^{Q}(k, R)$, whose multiplication is given by homological products.

Theorem 3.1. Let $Q$ be a standard graded $K$-algebra, $\varphi: Q \rightarrow R$ a surjective small homomorphism of graded $K$-algebras with $\operatorname{Ker} \varphi \neq 0$, and set $c=$ Rate $R$.

For every integer $i \geq 1$ there are then inequalities

$$
t_{i}^{Q}(R) \leq \operatorname{slope}_{Q} R \cdot i \leq i(c+1),
$$

and the following conditions are equivalent:

(i) $t_{i}^{Q}(R)=i(c+1)$.

(ii) $t_{h}^{Q}(R)=h(c+1)$ for $1 \leq h \leq i$.

(iii) $t_{1}^{Q}(R)=c+1$ and $\operatorname{Tor}_{i}^{Q}(k, R)_{i(c+1)}=\left(\operatorname{Tor}_{1}^{Q}(k, R)_{c+1}\right)^{i} \neq 0$. 
Before starting on the proof of the theorem we present an application, followed by a couple of easily verifiable sufficient conditions for the smallness of $\varphi$.

Corollary 3.2. With $J=\operatorname{Ker} \varphi$, the following assertions hold:

(1) $t_{i}^{Q}(R)=i(c+1)$ for some $i \geq 1$ implies $i \leq \operatorname{rank}_{k}\left(J / Q_{+} J\right)_{c+1}$.

(2) $t_{i}^{Q}(R)<i(c+1)$ holds for all $i>\operatorname{dim} Q-\operatorname{dim} R$ when $\operatorname{pd}_{Q} R$ is finite.

(3) $\operatorname{reg}_{Q} R \leq c \cdot \operatorname{pd}_{Q} R$.

Proof. Homological products are strictly skew-commutative for the homological degree, see [12, Ch. XI, §4], so $\left(\operatorname{Tor}_{1}^{Q}(k, R)_{*}\right)^{i}$ is the image of a canonical $k$-linear map

$$
\lambda_{i, *}: \bigwedge_{k}^{i}\left(J / Q_{+} J\right)_{*} \cong \bigwedge_{k}^{i} \operatorname{Tor}_{1}^{Q}(k, R)_{*} \rightarrow \operatorname{Tor}_{i}^{Q}(k, R)_{*} .
$$

(1) This follows from the map above and condition (iii) in Theorem 3.1.

(2) When $\operatorname{pd}_{Q} R$ is finite one has $\operatorname{grade}_{Q} R=\operatorname{dim} Q-\operatorname{dim} R$ by a theorem of Peskine and Szpiro [16], and $\lambda_{i, *}=0$ for $i>\operatorname{grade}_{Q} R$ from a theorem of Bruns [8]. Thus, Theorem 3.1 implies $\operatorname{Tor}_{i}^{Q}(k, R)_{j}=0$ for $j \geq i(c+1)$.

(3) The theorem gives $t_{i}^{Q}(R)-i \leq c i$ for each $i$, hence $\operatorname{reg}_{Q} R \leq c \cdot \operatorname{pd}_{Q} R$.

A bit of notation comes in handy at this point.

3.3. A standard graded $K$-algebra $R$ has a canonical presentation $R=\widetilde{R} / I_{R}$ with $\widetilde{R}$ the symmetric $K$ algebra on $R_{1}$ and $I_{R} \subseteq\left(\widetilde{R}_{4}\right)^{2}$, obtained from the epimorphism of $K$-algebras $\widetilde{R} \rightarrow R$ extending the identity map on $R_{1}$.

If $Q$ is a standard graded $K$-algebra and $\varphi: Q \rightarrow R$ is a surjective homomorphism with $\operatorname{Ker} \varphi \subseteq\left(Q_{+}\right)^{2}$, then $\widetilde{R} \rightarrow R$ factors as $\widetilde{R} \cong \widetilde{Q} \rightarrow Q \stackrel{\varphi}{\rightarrow} R$.

3.4. A homomorphism $\varphi$ as in 3.3 is small if $J=\operatorname{Ker} \varphi$ satisfies one of the conditions:

(a) $J \subseteq\left(f_{1}, \ldots, f_{a}\right)$, where $f_{1}, \ldots, f_{a}$ is some $Q$-regular sequence in $Q_{+}$.

(b) $J_{j}=0$ for $j \leq \operatorname{reg}_{\widetilde{Q}} Q$, where $Q=\widetilde{Q} / I_{Q}$ is the canonical presentation.

Indeed, see $[3,4.3]$ for (a), and Şega $[20,5.1,9.2(2)]$ for (b).

The hypothesis of Theorem 3.1 are in force for the rest of this section.

The proof of the theorem utilizes free resolutions with additional structure.

A model of $\varphi$ is a differential bigraded $Q$-algebra $Q[X]$ with the following properties: For every integer $n \geq 1$ there exists a $K$-linearly independent homogeneous subset $X_{n}=\{x \in X|| x \mid=n\}$, such that the underlying bigraded algebra is isomorphic to $Q \otimes_{K} \bigotimes_{n=1}^{\infty} K\left[X_{n}\right]$, where $K\left[X_{n}\right]$ is the exterior algebra of the graded $K$-vector space $K X_{n}$ when $n$ is odd, and the symmetric algebra of that space when $n$ is even. The differential satisfies $\operatorname{deg}(\partial(y))=\operatorname{deg}(y)$ for every element $y \in Q[X]$, and the following sequence of homomorphisms of free graded $Q$-modules is resolution of $R$ :

$$
\cdots \longrightarrow Q[X]_{n, *} \stackrel{\partial}{\longrightarrow} Q[X]_{n-1, *} \cdots \longrightarrow \cdots \longrightarrow Q[X]_{0, *} \longrightarrow 0
$$

A $Q$-basis of $Q[X]$ is provided by the set consisting of 1 and all the monomials $x_{1}^{d_{1}} \cdots x_{s}^{d_{s}}$ with $x_{r} \in X$, and with $d_{r}=1$ when $\left|x_{r}\right|$ is odd, respectively, $d_{r} \geq 1$ when $\left|x_{r}\right|$ is even. The model $Q[X]$ is said to be minimal if for each $x \in X$, the coefficient of every $x_{i} \in X$ in the expansion of $\partial(x)$ is contained in $Q_{+}$.

We summarize the properties of minimal models used in our arguments. 
3.5. A minimal model $Q[X]$ of $\varphi$ always exists, and is unique up to non-canonical isomorphism of differential bigraded $Q$-algebras; see [4, 7.2.4]. In such a model $\partial\left(X_{1}\right)$ is a minimal set of homogeneous generators of the $\operatorname{Ker} \varphi$ and $Q\left[X_{1}\right]$ is the Koszul complex on that set, with its standard bigrading, differential and multiplication.

3.6. Let $\widetilde{R}[Z]$ be a minimal model for the canonical presentation $\widetilde{R} \rightarrow R$, see 3.3 . Let $Z_{0}$ be a $K$-basis of $\widetilde{R}_{1}$, and choose a $k$-linearly independent set

$$
Z^{\prime}=\left\{z^{\prime}|| z^{\prime}|=| z \mid+1 \text { and } \operatorname{deg}\left(z^{\prime}\right)=\operatorname{deg}(z)\right\}_{z \in Z_{0} \sqcup Z} .
$$

By $[4,7.2 .6]$, there exists an isomorphism of bigraded $k$-vector spaces

$$
\operatorname{Tor}^{R}(k, k) \cong \bigotimes_{n=1}^{\infty} k\left\langle Z_{n}^{\prime}\right\rangle
$$

where $k\left\langle Z_{n}^{\prime}\right\rangle$ denotes the exterior algebra of the graded $k$-vector space $k Z_{n}^{\prime}$ when $n$ is odd, and the divided powers algebra of that space when $n$ is even.

3.7. Let $Q[X]$ be a minimal model for $\varphi$, and let $\widetilde{R} \stackrel{\psi}{\longrightarrow} Q \stackrel{\varphi}{\longrightarrow} R$ be a factorization of the canonical presentation $\widetilde{R} \rightarrow R$ as in 3.3. If $\widetilde{R}[Y]$ is a minimal model for $\psi$, then there is a minimal model $\widetilde{R}[Z]$ of $\widetilde{R} \rightarrow R$ with $Z=Y \sqcup X$; see [5, 4.11].

3.8. Proof of Theorem 3.1. For every integer $i \geq 2$ the following equality holds:

$$
t_{i-1}^{R}\left(R_{4}\right)-t_{0}^{R}\left(R_{4}\right)=t_{i}^{R}(k)-1 .
$$

Thus, for $i \geq 1$ the definition of slope and Proposition 1.4 applied with $M=k$ give

$$
t_{i}^{Q}(R) / i \leq \operatorname{slope}_{Q} R \leq c+1 .
$$

It remains to establish the equivalence of the conditions in the theorem.

(iii) $\Longrightarrow$ (ii). The condition $\left(\operatorname{Tor}_{1}^{Q}(k, R)_{c+1}\right)^{i} \neq 0$ forces $\left(\operatorname{Tor}_{1}^{Q}(k, R)_{c+1}\right)^{h} \neq 0$ for $h=1, \ldots, i$. As $\operatorname{Tor}^{Q}(k, R)$ is a bigraded algebra, one gets

$$
\operatorname{Tor}_{h}^{Q}(k, R)_{(c+1) h} \supseteq\left(\operatorname{Tor}_{1}^{Q}(k, R)_{c+1}\right)^{h} \neq 0 .
$$

This implies $t_{h}^{Q}(R) \geq(c+1) h$, and (3.8.2) provides the converse inequality.

(ii) $\Longrightarrow$ (i). This implication is a tautology.

(i) $\Longrightarrow$ (iii). The hypothesis means $\operatorname{Tor}_{i}^{Q}(k, R)_{i(c+1)} \neq 0$, so we have to prove

$$
\operatorname{Tor}_{i}^{Q}(k, R)_{i(c+1)}=\left(\operatorname{Tor}_{1}^{Q}(k, R)_{c+1}\right)^{i} .
$$

Let $Q[X] \rightarrow R$ be a minimal model and set $k[X]=k \otimes_{Q} Q[X]$. The bigraded $k$-algebras $\mathrm{H}(k[X])$ and $\operatorname{Tor}^{Q}(k, R)$ are isomorphic, with

$$
\operatorname{Tor}_{i}^{Q}(k, R)_{j} \cong \mathrm{H}_{i}(k[X])_{j} .
$$

In view of 3.7 each $x \in X_{n}$ can be viewed as an indeterminate of a minimal model of $\widetilde{R} \rightarrow R$, and so by 3.6 it defines an element $x^{\prime}$ in $\operatorname{Tor}_{n+1}^{R}(k, k)$ with $\operatorname{deg}(x)=\operatorname{deg}\left(x^{\prime}\right)$. From this equality and (3.8.1) we obtain

$$
\operatorname{deg}(x)=\operatorname{deg}\left(x^{\prime}\right) \leq t_{n+1}^{R}(k) \leq c n+1=c|x|+1 .
$$


The $k$-vector space $k[X]_{i,(c+1) i}$ has a basis of monomials $x_{1}^{d_{1}} \cdots x_{s}^{d_{s}}$ with $x_{r} \in X$ and $d_{r} \geq 1$. The following relations hold, with the inequality coming from (3.8.5):

$$
\begin{aligned}
\sum_{r=1}^{s} d_{r}\left|x_{r}\right| & =\left|x_{1}^{d_{1}} \cdots x_{s}^{d_{s}}\right|=i=(c+1) i-c i \\
& =\operatorname{deg}\left(x_{1}^{d_{1}} \cdots x_{s}^{d_{s}}\right)-c\left|x_{1}^{d_{1}} \cdots x_{s}^{d_{s}}\right| \\
& =\sum_{r=1}^{s} d_{r}\left(\operatorname{deg}\left(x_{r}\right)-c\left|x_{r}\right|\right) \\
& \leq \sum_{r=1}^{s} d_{r} .
\end{aligned}
$$

All $d_{r}$ and $\left|x_{r}\right|$ are positive integers, so for $1 \leq r \leq s$ we get first $\left|x_{r}\right|=1$, then $\left|x_{r}\right|=\operatorname{deg}\left(x_{r}\right)-c\left|x_{r}\right|$; that is, $\operatorname{deg}\left(x_{r}\right)=c+1$. We have now proved

$$
k[X]_{i,(c+1) i}=k\left[X_{1}\right]_{i,(c+1) i}=\left(k X_{1, c+1}\right)^{i} .
$$

The isomorphism (3.8.4) maps $\operatorname{Tor}_{1}^{Q}(k, R)_{c+1}$ to $k X_{1, c+1}$ and $\operatorname{Tor}_{i}^{Q}(k, R)_{(c+1) i}$ to a quotient of $k[X]_{i,(c+1) i}$, so the equalities above establish (3.8.3).

\section{Koszul algebras}

In this section we prove the theorem stated in the introduction.

Here $Q$ is a standard graded $K$-algebra, $\varphi: Q \rightarrow R$ a surjective homomorphism of graded $K$-algebras, and $M$ a graded $R$-module. As in [17], we say that $M$ is Koszul over $Q$ if $\operatorname{Tor}_{i}^{Q}(k, M)_{j}=0$ unless $i=j$. In the following proposition the Koszul hypotheses are related to the injectivity of $\operatorname{Tor}^{\varphi}(k, M)$ through the following lemma.

Proposition 4.1. Assume that $J$ is contained in $\left(Q_{+}\right)^{2}$.

(1) If $Q$ is Koszul, then $\varphi$ is small.

(2) If $\varphi$ is small and $M$ is Koszul over $Q$, then $\operatorname{Tor}^{\varphi}(k, M)$ is injective.

Proof. Forming vector space duals, one sees that the injectivity of $\operatorname{Tor}^{\varphi}(k, M)$ is equivalent to surjectivity of the homomorphism of bigraded $k$-vector spaces

$$
\operatorname{Ext}_{\varphi}(M, k): \operatorname{Ext}_{R}(M, k) \rightarrow \operatorname{Ext}_{Q}(M, k) .
$$

(1) For $M=k$ the map above is a homomorphism of $K$-algebras, with multiplication given by Yoneda products. The map $\operatorname{Ext}_{\varphi}^{1}(k, k)_{*}$ is isomorphic to

$$
\operatorname{Hom}_{R}\left(\varphi_{1}, k\right)_{*}: \operatorname{Hom}_{R}\left(R_{1}, k\right)_{*} \rightarrow \operatorname{Hom}_{Q}\left(Q_{1}, k\right)_{*},
$$

which is bijective as $J \subseteq\left(Q_{+}\right)^{2}$ holds. As $Q$ is Koszul, the $k$-algebra $\operatorname{Ext}_{Q}(k, k)$ is generated by $\operatorname{Ext}_{Q}^{1}(k, k)$, see [17, Ch. $2, \S 1$, Def. 1], so $\operatorname{Ext}_{\varphi}(k, k)$ is surjective.

(2) Yoneda products turn $\operatorname{Ext}_{\varphi}(M, k)$ into a homomorphism of bigraded left modules over $\operatorname{Ext}_{R}(k, k)$, with this algebra acting on $\operatorname{Ext}_{Q}^{0}(M, k)$ through $\operatorname{Ext}_{\varphi}(k, k)$. The bigraded module $\operatorname{Ext}_{Q}(M, k)$ is generated over $\operatorname{Ext}_{Q}(k, k)$ by $\operatorname{Ext}_{Q}^{0}(M, k)$, because $M$ is Koszul over $Q$; see [17, Ch. 2, $\S 1$, Def. 2]. Since $\varphi$ is small, $\operatorname{Ext}_{\varphi}^{0}(k, k)_{*}$ is surjective, and hence $\operatorname{Ext}_{Q}^{0}(M, k)$ generates $\operatorname{Ext}_{Q}(M, k)$ as an $\operatorname{Ext}_{R}(k, k)$-module as well. The map $\operatorname{Ext}_{\varphi}^{0}(M, k)_{*}$ is surjective, because it is canonically isomorphic to the identity map of $\operatorname{Hom}_{k}\left(M_{0}, k\right)_{*}$. It follows that $\operatorname{Ext}_{\varphi}(M, k)$ is surjective. 
4.2. Proof of the Main Theorem. Recall that $Q$ is Koszul, $J$ is a non-zero ideal of $Q$ with $J_{1}=0$, and $c=\operatorname{slope}_{R} R_{+}$. Note that $\varphi$ is small by Proposition 4.1(1).

(1) The inequality slope ${ }_{Q} R \leq c+1$ was proved as part of Theorem 3.1.

One has $t_{i}^{Q}(k)=i$ for $1 \leq i<\operatorname{pd}_{Q} k+1$ by the Koszul hypothesis on $Q$, and $t_{i}^{Q}(R) \geq i+1$ for $1 \leq i<\operatorname{pd}_{Q} R+1$ by the conditions $J_{1}=0$. The exact sequence

$$
\operatorname{Tor}_{i+1}^{Q}(k, k) \rightarrow \operatorname{Tor}_{i}^{Q}\left(k, R_{+}\right) \rightarrow \operatorname{Tor}_{i}^{Q}(k, R) .
$$

of graded vector spaces, which holds for every $i \geq 1$, therefore implies

$$
t_{i}^{Q}\left(R_{+}\right) \leq \max \left\{t_{i+1}^{Q}(k), t_{i}^{Q}(R)\right\}=t_{i}^{Q}(R),
$$

and hence slope $_{Q} R_{4} \leq \sup _{i \geqslant 1}\left\{\left(t_{i}^{Q}(R)-1\right) / i\right\}$. Now Proposition 1.2 gives

$$
c \leq \max \left\{\operatorname{slope}_{Q} R_{4}, \sup _{i \geqslant 1}\left\{\frac{t_{i}^{Q}(R)-1}{i}\right\}\right\} \leq \sup _{i \geqslant 1}\left\{\frac{t_{i}^{Q}(R)-1}{i}\right\} \leq \operatorname{slope}_{Q} R .
$$

When $\operatorname{pd}_{Q} R$ is finite the last inequality is strict, so one has $c<$ slope $_{Q} R$.

The inequalities in (2), (3), and (4) were proved as part of Corollary 3.2.

Finally, assume that $Q$ is a standard graded polynomial ring and $\operatorname{reg}_{Q} R=c p$ holds with $p=\operatorname{pd}_{Q} R$. Theorem 3.1 then shows that $\left(\operatorname{Tor}_{1}^{Q}(k, R)_{c+1}\right)^{p}$ is not zero, and so $\operatorname{Ker} \varphi$ needs at least $p$ minimal generators of degree $c+1$. As a bigraded $k$-algebra, $\operatorname{Tor}^{Q}(k, R)$ is isomorphic to the homology of the Koszul $E$ complex on some $K$-basis of $Q_{1}$, so one also has $\left(\mathrm{H}_{1}(E)\right)^{p} \neq 0$. Now a theorem of Wiebe, see [11, 2.3.15], implies that $\operatorname{Ker} \varphi$ is generated by a $Q$-regular sequence of $p$ elements.

Proposition 4.3. For a Koszul $K$-algebra $Q$ and $R=Q / J$ with $J \subseteq\left(Q_{+}\right)^{2}$ one has

$$
2 \leq \operatorname{slope}_{Q} R \leq \operatorname{slope}_{\widetilde{R}} R,
$$

where $R=\widetilde{R} / I_{R}$ is the canonical presentation. Equalities hold when $R$ is Koszul.

Proof. The canonical presentation factors as $\widetilde{R} \rightarrow Q \stackrel{\varphi}{\rightarrow} R$; see 3.3. Part (1) of the Main Theorem, applied to the homomorphism $\widetilde{R} \rightarrow Q$ and the $Q$-module $R$, gives inequalities $2 \leq \operatorname{slope}_{\widetilde{R}} Q \leq$ Rate $Q+1=2$, so Proposition 1.2 yields

$$
\operatorname{slope}_{Q} R \leq \max \left\{\operatorname{slope}_{\widetilde{R}} R, \operatorname{slope}_{\widetilde{R}} Q\right\}=\max \left\{\operatorname{slope}_{\widetilde{R}} R, 2\right\}=\operatorname{slope}_{\widetilde{R}} R .
$$

When $R$ is Koszul, the computation above gives $2 \leq \operatorname{slope}_{\widetilde{R}} R \leq$ Rate $R+1=2$.

\section{Slopes and Gröbner bases}

Let $R$ be a standard graded $K$-algebra and $R=\widetilde{R} / I_{R}$ its canonical presentation.

Let $T(R)$ denote the set of all term orders on all $K$-bases of $\widetilde{R}_{1}$. Letting $\operatorname{in}_{\tau}\left(I_{R}\right)$ denote the initial ideal corresponding to $\tau \in T$, Eisenbud, Reeves, and Totaro [14] set

$$
\Delta(R)=\inf _{\tau \in T(R)}\left\{t_{1}^{\widetilde{R}}\left(\widetilde{R} / \operatorname{in}_{\tau}\left(I_{R}\right)\right)\right\} .
$$

In words: $\Delta(R)$ is the smallest number $a$ such that $I_{R}$ has a Gröbner basis of elements of degree $\leq a$ with respect to a term order on some coordinate system. One has:

$$
\text { Rate } R \leq \Delta(R)-1,
$$


as announced by Backelin [7, Claim, p. 98] and proved in [14, Prop. 3]. We set

$$
\Delta^{\ell}(R)=\inf \{\Delta(Q)\},
$$

where $Q$ ranges over the set of all graded $K$-algebras satisfying $Q / L \simeq R$ for some ideal $L$ generated by a $Q$-regular sequence of elements of degree 1 .

Proposition 5.1. When $R$ is not a polynomial ring the following inequalities hold:

$$
2 \leq \text { Rate } R+1 \leq \Delta^{\ell}(R) \leq \Delta(R) .
$$

Proof. For $R \cong Q /(l)$ with $l$ a non-zero-divisor in $Q_{1}$, one has a chain

$$
\text { Rate } R=\operatorname{slope}_{R} R_{+}=\operatorname{slope}_{Q} R_{+}=\operatorname{slope}_{Q} Q_{+}=\text {Rate } Q \leq \Delta(Q)-1 .
$$

where the first and the last equalities hold by definition, the second one by Proposition $2.1(1)$, and the third one from the exact sequence $0 \rightarrow Q(-1) \rightarrow Q_{+} \rightarrow R_{+} \rightarrow 0$; the inequality, comes from (5.0.1). The second inequality in the proposition follows.

Combining the Main Theorem and the preceding proposition, one obtains:

Corollary 5.2. The following inequalities hold.

(1) $\operatorname{slope}_{\widetilde{R}} R \leq \Delta^{\ell}(R)$.

(2) $t_{i}^{\widetilde{R}}(R)<\Delta^{\ell}(R) \cdot i$ for all $i>\left(\operatorname{rank}_{K} R_{1}-\operatorname{dim} R\right)$.

(3) $\operatorname{reg}_{\widetilde{R}} R \leq\left(\Delta^{\ell}(R)-1\right) \cdot\left(\operatorname{rank}_{K} R_{1}-\operatorname{depth} R\right)$.

The research reported in this paper was prompted by the inequalities above, which were initially obtained by a very different argument; we proceed to sketch it.

5.3. For any isomorphism $R \simeq Q / L$, with $L$ generated by a regular sequence of linear forms, and for each $\tau \in T(Q)$ and every pair of integers $(i, j)$ one has:

$$
\beta_{i, j}^{\widetilde{R}}(R)=\beta_{i, j}^{\widetilde{Q}}(Q) \leq \beta_{i, j}^{\widetilde{Q}}\left(\widetilde{Q} / \operatorname{in}_{\tau}\left(I_{Q}\right)\right) ;
$$

see, for instance, $[9,3.13]$. The Taylor resolution of the monomial ideal $\operatorname{in}_{\tau}\left(I_{Q}\right)$, see $[15, \S 5]$, yields inequalities $t_{i}^{\widetilde{Q}}\left(\widetilde{Q} / \operatorname{in}_{\tau}\left(I_{Q}\right)\right) \leq t_{1}^{\widetilde{Q}}\left(\widetilde{Q} / \operatorname{in}_{\tau}\left(I_{Q}\right)\right) \cdot i$, which are strict for $i>\operatorname{rank}_{K} Q_{1}-\operatorname{dim} Q$. From these observations one obtains:

$$
\operatorname{slope}_{\widetilde{R}} R=\operatorname{slope}_{\widetilde{Q}} Q=\sup _{i \geqslant 1}\left\{t_{i}^{\widetilde{Q}}(Q) / i\right\} \leq \inf _{\tau \in T(Q)}\left\{t_{1}^{\widetilde{Q}}\left(\widetilde{Q} / \operatorname{in}_{\tau}\left(I_{Q}\right)\right)\right\}=\Delta(Q) .
$$

These inequalities imply part (1) of Corollary 5.2; part (3) is a formal consequence.

\section{Classes of quadratic algebras}

Let $R$ be a standard graded algebra. Several results proved above take the form of inequalities involving invariants of $R$. Here we examine relations between classes of algebras that are extremal with respect to such inequalities.

Following [13], we say that $R$ is G-quadratic if it satisfies $\Delta(R)=2$, and that it is $L G$-quadratic if $\Delta^{\ell}(R)=2$ holds. When one has slope $\widetilde{R}_{\widetilde{R}} R=2$ we say that $R$ is $S$-quadratic. The inequalities in (4.3.1) and (5.1.1) show that each one of these conditions means that some homological invariant takes the minimal possible value. They also establish the following hierarchy among the defining properties:

G-quadratic $\Longrightarrow$ LG-quadratic $\Longrightarrow$ Koszul $\Longrightarrow$ S-quadratic $\Longrightarrow$ quadratic 
We proceed to show that most of these implications are not invertible.

The first two examples are based on constructions of Roos.

Example 6.1. Let $P=K\left[x_{1}, x_{2}, x_{3}, x_{4}\right]$ be a polynomial ring and set

$$
R=P /\left(x_{1}^{2}, x_{2} x_{3}, x_{2}^{2}+x_{3} x_{4}, x_{1} x_{3}+x_{4}^{2}\right) .
$$

The $P$-module $R$ has a minimal free resolutions of the form

$$
0 \longrightarrow P^{3}(-7) \longrightarrow P^{7}(-6) \longrightarrow P^{6}(-4) \oplus P(-5) \longrightarrow P^{4}(-2) \longrightarrow P \longrightarrow 0 ;
$$

confer [19, Table 3, Case 12]. The resolution yields slope $\widetilde{R} R=5 / 2$.

Thus, not every quadratic algebra is S-quadratic.

To continue, we recall that for each graded $R$-module $M$ and every pair $(i, j)$ of integers a graded Betti number is defined by the formula

$$
\beta_{i, j}^{R}(M)=\operatorname{rank}_{k} \operatorname{Tor}_{i}^{R}(k, M)_{j},
$$

and that the Poincaré series of $M$ is the formal power series

$$
P_{M}^{R}(s, t)=\sum_{i \in \mathbb{N}, j \in \mathbb{Z}} \beta_{i, j}^{R}(M) s^{j} t^{i} \in \mathbb{Z}\left[s^{ \pm 1}\right] \llbracket t \rrbracket .
$$

Example 6.2. Let $P=K\left[x_{1}, x_{2}, x_{3}, x_{4}, x_{5}, x_{6}\right]$ be a polynomial ring.

For each integer $a \geq 2$ set $R=P / I$, where $I$ is the ideal

$\left(\left\{x_{i}^{2}\right\}_{1 \leq i \leq 6},\left\{x_{i} x_{i+1}\right\}_{1 \leq i \leq 5}, x_{1} x_{3}+a x_{3} x_{6}-x_{4} x_{6}, x_{1} x_{4}+x_{3} x_{6}+(a-2) x_{4} x_{6}\right)$.

When the characteristic of $K$ is zero, one has

$$
\text { slope }_{\widetilde{R}} R=2<2+(1 / a) \leq 1+\text { Rate } R \leq 2+(2 / a) .
$$

Thus, not every S-quadratic algebra is Koszul.

On the other hand, rates of non-Koszul algebras can be arbitrarily close to 1.

Proof of the inequalities. One has $t_{1}^{P}(R)=2$ because $I$ is generated by quadrics. The isomorphism $\operatorname{Tor}_{i}^{P}(k, R)_{j} \simeq \mathrm{H}_{i}\left(E \otimes_{P} R\right)_{j}$, where $E$ denotes the Koszul complex on some $K$-basis of $P_{1}$, and the equalities $R_{j}=0$ for $j \geq 3$ imply $t_{i}^{P}(R) \leq i+2$ for $2 \leq i \leq 6$. Comparing the numbers $t_{i}^{P}(R) / i$, one gets slope ${ }_{P} R=2$.

Set $H_{R}(s)=\sum_{j \geqslant 0} \operatorname{rank}_{k} R_{j} s^{j} \in \mathbb{Z} \llbracket s \rrbracket$. Roos $\left[18\right.$, Thm. $\left.1^{\prime}\right]$ proves the equalities

$$
H_{R}(s)=1+6 s+8 s^{2} \quad \text { and } \quad P_{k}^{R}(s, t)=\frac{1}{H_{R}(-s t)-(s t)^{a+1}(s+s t)} .
$$

Setting $h(s, t)=6-8 s t+s^{a+1} t^{a}+s^{a+1} t^{a+1}$, we get from here the expression

$$
P_{R_{+}}^{R}(s, t)=\frac{P_{k}^{R}(s, t)-1}{t}=\frac{s \cdot h(s, t)}{1-s t \cdot h(s, t)}=\sum_{i \geqslant 1} s^{i} t^{i-1} \cdot h(s, t)^{i} .
$$

Since $s^{a+2} t^{a}$ appears with a non-zero coefficient in the sum on the right, we get

$$
\operatorname{slope}_{R}\left(R_{4}\right) \geq(a+1) / a=1+(1 / a) .
$$

Following [2], for each $f(s, t)=\sum_{i, j \geqslant 0} b_{i, j} s^{j} t^{i} \in \mathbb{R}[s] \llbracket t \rrbracket$ we set

$$
\operatorname{rate}(f(s, t))=\sup _{i, j}\left\{j / i \mid i \geq 1 \text { and } b_{i, j} \neq 0\right\} .
$$


Now $[2,1.1]$ gives the inequality in the following chain:

$$
\begin{aligned}
\operatorname{slope}_{R}\left(R_{+}\right) & =\operatorname{rate}\left(\frac{s \cdot h(s, t)}{1-s t \cdot h(s, t)}\right) \\
& \leq \max \{\operatorname{rate}(s \cdot h(s, t)), \operatorname{rate}(1-s t \cdot h(s, t))\} \\
& =\max \left\{\frac{a+2}{a}, \frac{a+2}{a+1}\right\} \\
& =1+(2 / a) .
\end{aligned}
$$

The desired inequalities follow, as one has Rate $R=\operatorname{slope}_{R}\left(R_{4}\right)+1$ by definition,

Example 6.3. In a discussion at the end of [14], the authors show that the algebra

$$
R=k\left[x_{1}, x_{2}, x_{3}\right] /\left(x_{1}^{2}+x_{1} x_{2}, x_{2}^{2}+x_{2} x_{3}, x_{3}^{2}+x_{3} x_{1}\right)
$$

is complete intersection and is not G-quadratic. On the other hand, Caviglia has observed that every complete intersection of quadrics is LG-quadratic; see [13, 1.4].

Thus, not every LG-quadratic algebra is G-quadratic.

The preceding examples leave us with:

Question 6.4. Is every Koszul algebra LG-quadratic?

The Betti numbers $\beta_{i}^{\widetilde{R}}(R)=\sum_{j \in \mathbb{Z}} \beta_{i, j}^{\widetilde{R}}(R)$ might help separate the two notions.

Indeed, when $R$ is LG-quadratic one has $R \cong Q / L$ and $Q=\widetilde{Q} / I_{Q}$, where $Q$ is a standard graded $K$-algebra, $L$ is an ideal generated by a $Q$-regular sequence of linear forms, and the initial ideal $\operatorname{in}_{\tau}\left(I_{Q}\right)$ for some $\tau \in T(Q)$ is generated by quadrics. As a consequence, one has $\beta_{1}^{\widetilde{R}}(R)=\beta_{1}^{\widetilde{Q}}(Q)=\beta_{1}^{\widetilde{Q}}\left(\widetilde{Q} / \operatorname{in}_{\tau}\left(I_{Q}\right)\right)$, so we obtain

$$
\beta_{i}^{\widetilde{R}}(R) \leq \beta_{i}^{\widetilde{Q}}\left(\widetilde{Q} / \operatorname{in}_{\tau}\left(I_{Q}\right)\right) \leq\left(\begin{array}{c}
\beta_{1}^{\widetilde{Q}}\left(\widetilde{Q} / \operatorname{in}_{\tau}\left(I_{Q}\right)\right) \\
i
\end{array}\right)=\left(\begin{array}{c}
\beta_{1}^{\widetilde{R}}(R) \\
i
\end{array}\right)
$$

with inequalities coming from (5.3.1) and the Taylor resolution. Thus, we ask:

Question 6.5. If $R$ is a Koszul algebra, does $\beta_{i}^{\widetilde{R}}(R) \leq\left(\begin{array}{c}\beta_{1}^{\widetilde{R}}(R) \\ i\end{array}\right)$ hold for every $i$ ?

\section{References}

[1] D. J. Anick, On the homology of associative algebras, Trans. Amer. Math. Soc. 296 (1986), 641-659.

[2] A. Aramova, Ş. Bărcănescu, J. Herzog, On the rate of relative Veronese submodules, Rev. Roumaine Math. Pures Appl. 40 (1995), 243-251.

[3] L. L. Avramov, Small homomorphisms of local rings, J. Algebra 50 (1978), 400-453.

[4] L. L. Avramov, Infinite free resolutions, Six lectures on commutative algebra (Bellaterra, 1996), Progr. Math. 166, Birkhuser, Basel, 1998; 1-118.

[5] L. L. Avramov, S. Iyengar, André-Quillen homology of algebra retracts, Ann. Sci. École Norm. Sup. (4) 36 (2003), 431-462.

[6] L. L. Avramov, I. Peeva, Finite regularity and Koszul algebras, Amer. J. Math. 123 (2001), $275-281$

[7] J. Backelin, On the rates of growth of the homologies of Veronese subrings, Algebra, algebraic topology, and their interactions (Stockholm, 1983), Lecture Notes in Math. 1183, Springer, Berlin, 1986; 79-100.

[8] W. Bruns, On the Koszul algebra of a local ring, Illinois J. Math. 37 (1993), 278-283. 
[9] W. Bruns, A. Conca, Gröbner bases and determinantal ideals, Commutative algebra, singularities and computer algebra (Sinaia, 2002), NATO Sci. Ser. II Math. Phys. Chem., 115, Kluwer Acad. Publ., Dordrecht, 2003; 9-66.

[10] W. Bruns, A. Conca, T. Römer, Koszul homology and syzygies of Veronese subalgebras, arXiv:0902.2431.

[11] W. Bruns, J. Herzog, Cohen-Macaulay rings, Revised edition, Cambridge Studies Adv. Math. 39, University Press, Cambridge, 1998.

[12] H. Cartan, S. Eilenberg, Homological algebra, Princeton Univ. Press, Princeton, NJ, 1956.

[13] A. Conca, Koszul algebras and Gröbner bases of quadrics, Proceedings of the 29th Symposium on Commutative Algebra in Japan, Nagoya, Japan, 2007; 127-133; arXiv:0903.2397.

[14] D. Eisenbud, A. Reeves, B. Totaro, Initial ideals, Veronese subrings, and rates of algebras, Adv. Math. 109 (1994), no. 2, 168-187.

[15] R. Fröberg, Some complex constructions with applications to Poincaré series, Séminaire d'Algèbre Paul Dubreil, (Paris, 1977-1978), Lecture Notes in Math., 740, Springer, Berlin, 1979; 272-284.

[16] C. Peskine, L. Szpiro, Syzygies et multiplicités, C. R. Acad. Sci. Paris Sér. A 278 (1974), 1421-1424.

[17] A. Polishchuk, L. Positselski, Quadratic algebras, Univ. Lecture Ser. 37, Amer. Math. Soc., Providence, RI, 2005.

[18] J.-E. Roos, Commutative non Koszul algebras having a linear resolution of arbitrarily high order. Applications to torsion in loop space homology, C. R. Acad. Sci. Paris 316 (1993), 11231128.

[19] J.-E. Roos, A description of the homological behaviour of families of quadratic forms in four variables, Syzygies and Geometry (Boston 1995), Northeastern Univ. 1995; pp. 86-95.

[20] L. M. Şega, Homological properties of powers of the maximal ideal of a local ring, J. Algebra 241 (2001), 827-858.

Department of Mathematics, University of Nebraska, Lincoln, NE 68588, U.S.A.

E-mail address: avramov@math.unl.edu

Dipartimento di Matematica, Universita di Genova, Via Dodecaneso 35, I-16146 Genova, ITALY

E-mail address: conca@dima.unige.it

Department of Mathematics, University of Nebraska, Lincoln, NE 68588, U.S.A.

E-mail address: iyengar@math.unl.edu 